\title{
BMJ Statin use and asthma control Open in patients with severe asthma
}

Amir A Zeki, ${ }^{1-4}$ Justin Oldham, ${ }^{1}$ Machelle Wilson, ${ }^{5}$ Olga Fortenko, ${ }^{1}$ Vishal Goyal, ${ }^{1}$ Michael Last, ${ }^{5}$ Andrew Last, ${ }^{6}$ Ayan Patel, ${ }^{3}$ Jerold A Last, ${ }^{1,2,4}$ Nicholas J Kenyon ${ }^{1-4}$

To cite: Zeki AA, Oldham J, Wilson $M$, et al. Statin use and asthma control in patients with severe asthma. BMJ Open 2013;3: e003314. doi:10.1136/ bmjopen-2013-003314

- Prepublication history and additional material for this paper is available online. To view these files please visit the journal online (http://dx.doi.org/10.1136/ bmjopen-2013-003314).

Received 29 May 2013

Revised 4 July 2013

Accepted 5 July 2013

For numbered affiliations see end of article.

\footnotetext{
Correspondence to Dr Amir A Zeki; amir.zeki@ucdmc.ucdavis. edu
}

\begin{abstract}
Objectives: We hypothesised that severe asthmatics taking a statin drug, in addition to inhaled corticosteroids/long-acting $\beta$-agonist inhaler therapy, would have better asthma symptom control and improved lung function compared to their controls.
\end{abstract}

Study design: A retrospective, cross-sectional study of 165 patients with severe asthma seen from 2001-2008. Hierarchical linear and logistic regression models were used for modelling fitting.

Setting: University of California, Davis Medical Center (Sacramento, California, USA). Academic, single-centre, severe asthma subspecialty clinic.

Participants: 612 screened, 223 eligible and 165 adult patients were included in the final study ( $\mathrm{N}=165 ; 31$ statin users and 134 non-users).

Primary and secondary outcome measures: The primary endpoint was asthma control as measured by the Asthma Control Test (ACT). The secondary endpoints included lung function, symptoms and the need for corticosteroid burst and peripheral eosinophil count.

Results: At baseline, statin users compared to nonusers were older, had lower lung function ( $F E V_{1} \%$ predicted, $\mathrm{FEV}_{1}$, forced vital capacity and $\mathrm{FEF}_{25-75 \%}$ ) and had a higher prevalence of comorbid conditions. Statin use was associated with more aspirin and ipratropium inhaler use than in non-users. Patients in both groups were obese (body mass index $\geq 30$ ). Statin users had better asthma symptom control compared to non-users (higher adjusted mean ACT score by $2.2 \pm 0.94$ points, $p<0.02)$. Median statin use was for 1 year. There were no statistically significant differences in lung function, corticosteroid or rescue bronchodilator use or peripheral eosinophilia between the two groups.

Conclusions: In our severe asthma referral population, statin users already taking inhaled controller therapy achieved better asthma control compared to non-users. The implications of this study is that patients with severe asthma could potentially benefit from added statin treatment. Because our study population was on average obese, the obese severe asthmatic may be a viable asthma subphenotype for further studies. Prospective randomised clinical trials evaluating the safety and efficacy of statins in severe asthma are warranted.

\section{INTRODUCTION}

Asthma remains difficult to control and can progress in subsets of patients. Patients with

\section{ARTICLE SUMMARY}

Article focus

- Does adding a statin drug to standard-of-care inhaled corticosteroids and long-acting $\beta$-agonists have any therapeutic benefit in a real-life cohort of severe asthma?

- We hypothesise that severe asthmatics would benefit from statin therapy and have improved asthma control.

Key messages

- Patients with severe asthma who take a statin drug in addition to standard inhaler controller therapy have improved asthma symptoms. Specifically, the improved Asthma Control Test score associated with statin use predicts a reduced risk of acute exacerbations and reduced need for rescue bronchodilator use. Thus, statin use is associated with improved control of severe asthma.

- Therapies for severe asthma are lacking, a subpopulation of asthma which comprises $10 \%$ yet incurs over $80 \%$ of asthma healthcare costs, are lacking. The widely available statin drugs could be used to treat this difficult-to-control group, which would have a considerable public health impact worthy of further study.

- The obese patient with severe asthma may be a viable asthma subphenotype to study the potential therapeutic benefit of statins.

Strengths and limitations of this study

- Utilisation of a real-life, decade-long severe asthma population on maximal controller therapy.

- Median duration of statin use of 1 year.

- Novel positive association and long-term correlation between statin use and severe asthma disease control.

- The study design is retrospective, cross-sectional and observational.

- The database includes a relatively small sample size of statin users.

severe asthma represent approximately $10 \%$ of the asthma population, ${ }^{1}$ who have the lowest quality of life and incur the greatest healthcare costs. Innovative therapies are urgently needed. One avenue of interest is in the statin drugs (or 'statins') which inhibit 3-hydroxy-3methyl-glutaryl-coenzyme A reductase (HMGcoenzyme A reductase or HMGCR), the first 
and rate-limiting step in cholesterol biosynthesis. Epidemiological data have linked statin use with improvements in lung health and asthma-related hospitalisations and/or emergency room visits, in particular in asthma and chronic obstructive pulmonary disease $(\mathrm{COPD}) .^{2}{ }^{3}$ Improvements in exacerbation rates, intubation rates and lung function as measured by $\mathrm{FEV}_{1}$ and forced vital capacity (FVC) have been reported. ${ }^{4} 5$ Animal experiments and in vitro studies also support the potential antiinflammatory, antiproliferative, antioxidant and immunomodulatory effects of the statin drugs. ${ }^{6-8}$

Until now, seven clinical trials in patients with mild to moderate allergic asthma have failed to confirm a benefit to statins with respect to symptom control or lung function. ${ }^{9-15}$ Although four of these trials measured indices of asthma control, ${ }^{9-12}$ none of them measured exacerbations and none exclusively evaluated severe asthmatics. ${ }^{9-15}$ However, in asthmatics who smoke, atorvastatin added to an inhaled corticosteroid (ICS) improved their asthma quality of life, suggesting that some but not all patients with asthma might benefit from statins. ${ }^{10}$ Several ongoing clinical trials are currently investigating various statins in asthma and COPD.

Given that severe asthma is refractory to many treatments, is difficult to manage and can progress to irreversible airflow obstruction akin to COPD, ${ }^{16}$ we investigated whether statin use might confer a benefit. In a retrospective cross-sectional study of 165 patients with severe asthma in our University of California, Davis Asthma Network (UCAN) clinic, we investigated whether statin use was associated with improvement in asthma control and/or lung function. We hypothesised that severe asthmatics taking a statin drug, in addition to ICS \pm long-acting $\beta$-agonist (LABA) inhaler therapy, would have better asthma symptom control and improved lung function compared to controls. We found that statin use was associated with higher Asthma Control Test (ACT) scores (ie, better symptom control), with no significant differences in lung function or peripheral eosinophilia. We could not evaluate the effects on hospitalisation or other severe asthma exacerbations, since the incidence of these outcomes in our UCAN population is very low (see online supplement for more information and additional references), precluding adequate statistical power to detect a difference.

This study highlights important considerations for future planned asthma-statin clinical intervention trials given asthma's considerable phenotypic heterogeneity. The potential therapeutic role of statins in severe asthma remains an open question worthy of further study.

\section{MATERIALS AND METHODS}

Materials and methods are shown in online supplemental material (for additional details).

\section{Study design}

We conducted a retrospective, cross-sectional study of patients with severe asthma at the UCAN clinic from February 2001 to November 2008.

The study spanned the years of transition at our institution from paper medical records to the electronic medical record (EMR). The process of identifying and reviewing the older paper records took longer than initially expected. On the basis of our initial Institutional Review Board (IRB) application, we decided to finish our data collection in the upcoming full calendar year, which was 2008.

\section{Study subjects}

A total of 612 patients were screened from 2001-2008 in our UCAN database and hospital EMR. On the basis of the eligibility criteria, a total of 165 patients with severe asthma were evaluated: 31 statin users (exposure group) and 134 non-users (control group; figure 1).

Inclusion criteria included age $\geq 18$ years, objective findings of asthma that meet the definition of severe asthma by the American Thoracic Society (ATS) statement on refractory asthma (2000) and Global Initiative for Asthma (GINA) 2007 criteria, on daily chronic moderate-to-high dose ICSs and taking a statin drug, with two or more qualified visits in the UCAN clinic. Statin use during the study period was confirmed by reviewing the EMR and/or pharmacy refill records and/ or change in serum fasting lipids. Atopic status was determined by measuring the blood IgE levels and radioallergosorbent test (RAST) panel.

Exclusion criteria included COPD or emphysema, asthma-COPD overlap syndrome, previous lung radiation, toxic inhalational lung injury, interstitial lung disease or other chronic inflammatory airway disorder(s) (eg, bronchiectasis, cystic fibrosis and chronic infectious bronchitis); inadequate objective documentation of

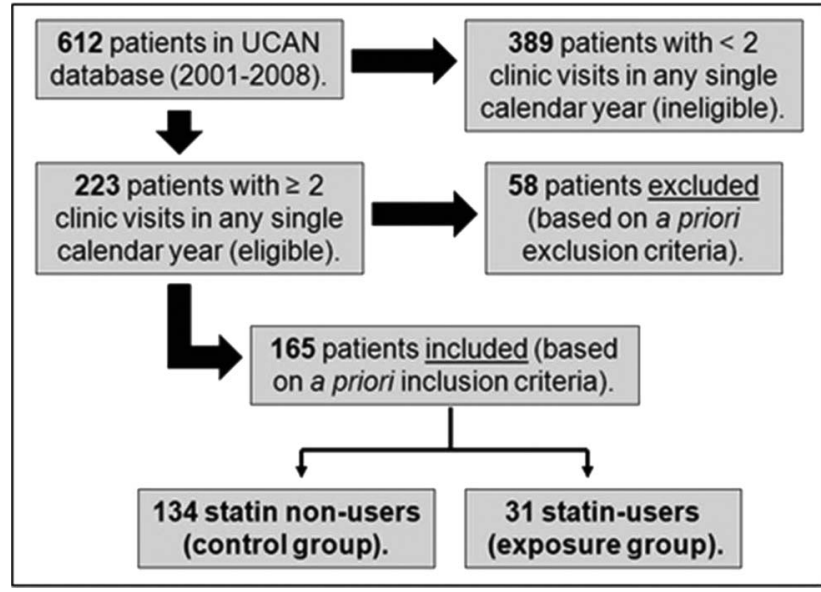

Figure 1 Flow diagram of patient selection displaying the total number of participants screened, the eligibility criteria and the number of participants who were included and excluded. 
asthma diagnosis; statin non-adherence or statin use documented in only one study visit; and statin adverse reaction or allergy.

The diagnosis of asthma and the exclusion of COPD (and other respiratory diseases) were based on clinical criteria as determined by the attending pulmonary asthma specialist and as per above. These clinical parameters included but were not limited to the following: history and physical examination, pulmonary function test (PFT) and/or spirometry, relevant laboratory tests and thoracic imaging, including chest X-ray and/or CT, where available.

\section{Study size}

We powered this study to find between-group differences that are similar in magnitude to that observed in the Hothersall $e t a l^{12}$ study. Using the sputum macrophage data to estimate the mean and $\mathrm{SD}$, with a two-sided $\alpha$ of 0.05 , power of $80 \%$ and a $3: 1$ ratio of non-statin user to statin user, we calculated a total sample size of $\mathrm{N}=122$, of which 31 were statin users. These sample size calculations were made using the Two-Arm Normal sample size calculator on the Southwest Oncology Group website http://www.swogstat.org/stat/public/two_normal.htm.

We selected the sputum macrophage as an indicator of the statin's anti-inflammatory effect, which we assumed might translate into a clinical effect. These were the best published data available at the time we made our power analysis. Given the large number of patients who qualified for the study, in the end we studied 134 statin non-users and 31 statin users for a total sample size of 165 , which is a significantly higher sample size than originally estimated (ie, $\mathrm{N}=165$ vs 122).

\section{Data collection procedures}

Data were collected on eligible patients who were followed in UCAN for at least 1 year. All patients seen in the UCAN clinic during the study time frame were initially evaluated for eligibility using EMR and those patients with less than two qualified visits were deemed ineligible (figure 1). The remaining patients' paper charts were also reviewed for inclusion and exclusion criteria.

Relevant comorbid diseases, asthma symptoms, medications, patient-reported peak expiratory flow rate (PEFR) and in-clinic spirometry were documented by respiratory therapists prior to evaluation by the pulmonologist staffing the clinic. The severity of asthma and need for systemic steroids were determined by the staff pulmonologist. Statin use had to be documented at least once during any clinic visit to designate the patient as a 'statin user.' The majority of patients had 'statin use' documented in every eligible visit included in the study.

\section{Variables, predictors and endpoints}

The main predictor variable or exposure was statin use and our a priori primary endpoint was the ACT score, a validated and recommended tool to identify and quantify patients' asthma control. ${ }^{17-19}$ We chose ACT in lieu of severe exacerbations as the primary endpoint for two reasons: (1) the clinical utility of ACT scores in managing asthma compared to other objective parameters and (2) the low number of severe exacerbations in our management programme UCAN cohort $(<8 \%)$, which limited statistical power and our ability to detect a difference between the two groups. The effect of statins on severe exacerbations is reported in the online supplementary material.

The a priori secondary endpoints included the following: (1) lung function $\left(\mathrm{FEV}_{1}, \mathrm{FVC}, \mathrm{FEF}_{25-75 \%}\right.$ and PEFR), (2) symptoms (need for corticosteroid burst, short-acting bronchodilator use (albuterol and ipratropium), limited physical activity and nocturnal symptoms) and (3) peripheral eosinophil count.

If the prebronchodilator and postbronchodilator lung function values were available, both were recorded, but the post bronchodilator test with the highest value was used for all analyses. A change of $>20 \%$ improvement in PEFR from baseline or predicted value was considered as a clinically significant indicator of airflow variability.

\section{Statistical analyses}

\section{Summary statistics and epidemiology}

To obtain summary statistics for the various covariates of interest, variables measured at each encounter (ie, multiple encounters per patient) were averaged across encounters to obtain one value per variable for each patient. To test for differences between statin users and non-users, independent sample t tests were performed when the variable was continuous and approximately normally distributed. The Kruskal-Wallis test was performed for the proportions of encounters that were not normally distributed. The $\chi^{2}$ or Fisher's exact test was performed when the proportions observed were all either 0 or 1 .

\section{Model selection}

A multivariate model selection approach was implemented to test for effects due to statin use, where we used a backward selection procedure to identify significant confounders. For continuous endpoints, a hierarchical linear model with a random intercept was fitted using the SAS software procedure Proc Mixed. For binary endpoints, a hierarchical logistic regression with a random intercept was fitted using the SAS software procedure Proc Glimmix. This approach accounts for the intersubject correlation due to the multiple encounters (ie, clinic visits) for each patient.

To control for possible confounders, tests for differences between statin users and non-users were conducted on the pertinent variables, and any variable with $\mathrm{p} \leq 0.1$ was included during the model selection procedure. To be conservative, we chose the cut-off $p$ value of 0.1 to avoid eliminating possible confounders, while at the same time maintaining power by not including unnecessary variables. A multivariate model selection 
approach was implemented to test for effects due to statin use. We used a backward selection procedure to identify significant confounders. In the first stage, all variables where a significant difference was observed between statin users and non-users were included in the model, but statin use was not included at this initial stage. Additionally, variables known to be important in the published literature, whether or not significant differences occurred between the two groups, were also included in the models (eg, ICS and LABA use, systemic corticosteroid use, smoking, body mass index (BMI)/ obesity, ethnicity, exacerbations in the previous 12 months and $F E V_{1} \%$ predicted). For each endpoint, the initial stage of model selection included all such possible confounders as described above. At each step, the variable with the highest non-significant $p$ value was removed until all of the remaining variables were significant at the 0.1 level. In the second stage, the treatment variable (statin user vs non-user) and its interaction with all significant covariates were included in the model. In the next stage, non-significant interaction terms were eliminated using backward selection as described above. This procedure tests for significant effects due to statin use after controlling for covariates for which the data provide evidence of possible confounding effects. Our statistical modelling utilised a two-way $\alpha$ to account for positive and negative associations with statin use with a final $p$ value of $<0.05$ to indicate statistical significance (table 1).

\section{RESULTS}

The results are shown in online supplemental material (for additional details).

\section{Cohort characteristics}

Summary statistics of patient demographics and characteristics, as well as the types of statins used, are shown in tables $2-4$. In general, statin users were older $(60.8 \pm 11.7$ vs $46.6 \pm 13.9$ years, $\mathrm{p}=0.0001$ ); had lower lung function as measured by $F E V_{1} \%$ predicted $(67.5 \pm 21.9 \%$ vs 75.5 $\pm 21.2 \%, \quad \mathrm{p}=0.07), \quad \mathrm{FEV}_{1} \quad(1.81 \pm 0.68$ vs $2.22 \pm 0.82 \mathrm{~L}$, $\mathrm{p}=0.0052)$, FVC $(2.56 \pm 0.82$ vs $3.02 \pm 0.99 \mathrm{~L}, \mathrm{p}=0.0085)$ and $\mathrm{FEF}_{25-75 \%}(1.47 \pm 0.90$ vs $1.95 \pm 1.07 \mathrm{~L}, \mathrm{p}=0.0125)$; had a higher prevalence of coronary artery disease (CAD, $16.1 \%$ vs $0 \%$; $<0.0001$ ), hyperlipidaemia (HPL, 93.6\% vs $9.5 \% ; \mathrm{p}<0.0001)$ and diabetes mellitus $(32.3 \%$ vs $13.4 \%, \mathrm{p}=0.012$ ) and a trend towards a higher prevalence of gastro-oesophageal reflux disease $(83.9 \%$ vs $64.9 \%, \mathrm{p}=0.41)$; had a lower prevalence of sinusitis $(0 \%$ vs $14.2 \%, \mathrm{p}=0.026)$ and a higher medication use of ipratropium $(48.4 \%$ vs $31.3 \%, \mathrm{p}=0.023)$ and aspirin $(25.8 \%$ vs $11.2 \%, \mathrm{p}=0.031)$. There were no statistically significant differences in sex, ethnicity, BMI, weight, PEFR, ACT score and peripheral blood counts, including eosinophils, atopic status, smoking history, prevalence of rhinosinusitis or medication use of the following drugs: ICS, LABA, albuterol, systemic corticosteroids, montelukast, 5-lipoxygenase inhibitor, omalizumab, non-steroidal antiinflammatory drugs, proton-pump inhibitor or histamine receptor-2 $\left(\mathrm{H}_{2}\right)$ blockers.

\section{Asthma control}

Statin users had a significantly higher adjusted mean ACT score by $2.2 \pm 0.94$ points $(\mathrm{p}=0.02)$, indicating better asthma control among statin users compared to non-users (table 1). The final model showed that statin use, smoking history, obesity $(\mathrm{BMI} \geq 30)$, gastrooesophageal reflux disease (GERD) and FVC predict ACT score. Smoking was associated with a reduction in ACT of $4.04 \pm 1.2 \quad(p=0.0013)$, obesity with a reduction of $2.1 \pm 0.78(p=0.0094)$ and GERD with a reduction of 2.6 $\pm 0.96(p=0.0072)$. Conversely, for each $1 \mathrm{~L}$ increase in FVC, the ACT score increased by 2.2 $\pm 0.41 \quad(p<0.0001)$. There were no significant interactions among significant variables.

Our analysis also shows that severe asthmatics taking statins had higher adjusted mean ACT scores across the spectrum of comorbid conditions. For example, for patients without comorbid obesity, GERD, or smoking history, statin users had an ACT score of 20.7 (the highest adjusted mean ACT score) and non-users a score of 18.5 ( $p=0.02$ ). For patients with all three conditions, statin users had an ACT score of 11.9 and non-users a score of 9.8 (the lowest adjusted mean ACT score, $\mathrm{p}=0.02$; figure 2).

The range of ACT scores indicates relative asthma control or lack thereof. ${ }^{17}$ ACT scores $\geq 20$ indicate "wellcontrolled' asthma, 19-16 indicate 'somewhat controlled' asthma, and $\leq 15$ indicate 'poorly controlled' asthma. Specifically, for patients with a history of smoking and independently for those who are obese with GERD, statin use was associated with better control by ACT criteria from poorly controlled $(\leq 15)$ to somewhat controlled (16-19) asthma (figure 2). For patients without obesity, GERD or smoking history, statin use improved them from somewhat controlled (16-19) to well-controlled ( $\geq 20)$ asthma (figure 2). These adjusted mean ACT values were based on our statistical model using a mean FVC of $2.94 \mathrm{~L}$, the mean value in our study population.

\section{Lung function}

For the secondary endpoint of lung function, we evaluated four parameters: $\mathrm{FEV}_{1}, \mathrm{FVC}, \mathrm{FEF}_{25-75 \%}$ and PEFR (table 1). Trends favoured statin use, but none were statistically significant in the final model.

\section{Symptoms}

To assess the effect of statins on asthma symptoms, we evaluated five variables as indicators of asthma impairment and risk for future exacerbations, including the need for oral corticosteroid burst, bronchodilator use (albuterol and ipratropium) and activity of daily living symptoms (limited physical activity and nocturnal awakenings; table 1). 
Table 1 Statin effect sizes adjusted for confounders

\begin{tabular}{|c|c|c|c|c|}
\hline & $\begin{array}{l}\text { Statin effect } \\
\text { size }\end{array}$ & p Value* & Favours & Final model \\
\hline \multicolumn{5}{|l|}{ Primary endpoint† } \\
\hline$\Delta$ Adjusted mean ACT score & $2.2 \pm 0.94$ & 0.02 & $\begin{array}{l}\text { Statin } \\
\text { user }\end{array}$ & Statin use, obesity, GERD, h/o smoking and FVC predict ACT score \\
\hline \multicolumn{5}{|l|}{ Secondary endpoints } \\
\hline \multicolumn{5}{|l|}{ Lung function† } \\
\hline$\Delta \mathrm{FEV}_{1}(\mathrm{~L})$ & $0.036 \pm 0.06$ & 0.56 & $\begin{array}{l}\text { Statin } \\
\text { user }\end{array}$ & Age, FEV1\% predicted and FVC predict FEV1 \\
\hline$\Delta \mathrm{FVC}(\mathrm{L})$ & $0.026 \pm 0.18$ & 0.88 & $\begin{array}{l}\text { Statin } \\
\text { user }\end{array}$ & Age and ethnicity predict FVC \\
\hline$\Delta \mathrm{FEF}_{25-75 \%}(\mathrm{~L})$ & $0.088 \pm 0.16$ & 0.59 & $\begin{array}{l}\text { Statin } \\
\text { user }\end{array}$ & Age, FEV1\% predicted, FVC and montelukast predict $\mathrm{FEF}_{25-75 \%}$ \\
\hline$\triangle$ PEFR (L/min) & $-26.8 \pm 25.7$ & 0.92 & Non user & Systemic steroid use, PPI or $\mathrm{H}_{2}$-blockers, and FVC predict PEFR \\
\hline \multicolumn{5}{|r|}{ ( } \\
\hline Corticosteroid burst & 0.93 & 0.84 & $\begin{array}{l}\text { Statin } \\
\text { user }\end{array}$ & FEV1\% predicted, h/o smoking and DM predict the need for a steroid burst in clinic \\
\hline Albuterol inhaler use & 0.46 & 0.41 & $\begin{array}{l}\text { Statin } \\
\text { user }\end{array}$ & ICS use strongly predicts albuterol inhaler use \\
\hline Ipratropium inhaler use & 0.83 & 0.71 & $\begin{array}{l}\text { Statin } \\
\text { user }\end{array}$ & Age, h/o smoking, and FVC predict ipratropium inhaler use \\
\hline Limited physical activity & 1.09 & 0.91 & Non user & Aspirin use and FVC predict limited physical activity \\
\hline Nocturnal symptoms & 1.71 & 0.39 & Non user & Obesity predicts nocturnal symptoms/awakening \\
\hline \multicolumn{5}{|l|}{ Peripheral blood counts† } \\
\hline$\Delta$ Total WCC $\left(\mathrm{K} / \mathrm{mm}^{3}\right)$ & $-0.77 \pm 0.72$ & 0.29 & - & Systemic steroid use predicts change in WCC \\
\hline$\Delta$ Absolute eosinophil count & $0.024 \pm 0.06$ & 0.68 & Non user & $\begin{array}{l}\text { No variable in the model predicts the change in absolute eosinophil count (see online } \\
\text { supplement) }\end{array}$ \\
\hline$\Delta \%$ Eosinophil count & $0.28 \pm 0.64$ & 0.66 & Non user & Systemic corticosteroid and aspirin use predict the \% eosinophil count \\
\hline \multicolumn{5}{|c|}{$\begin{array}{l}\text { This symbol represents 'delta' or the change due to the statin's effect, hence, 'statin effect size'. } \\
\text { *p Values represent the significance of statin use in the final model. } \\
\text { †Statin effect sizes are listed as the model estimate } \pm \text { SE. ACT was adjusted for the confounders of obesity, GERD, h/o smoking and FVC. } \\
\text { fStatin effect sizes are listed as ORs. } \\
\text { ACT, Asthma Control Test questionnaire; DM, diabetes mellitus; FVC, forced vital capacity; GERD, gastro-oesophageal reflux disease; h/o, history of; H, histamine receptor-2; ICS, inhalec } \\
\text { corticosteroid; PEFR, peak expiratory flow rate; PPI, proton-pump inhibitor; WCC, white cell count. }\end{array}$} \\
\hline
\end{tabular}


Table 2 Study population unadjusted summary statistics

\begin{tabular}{|c|c|c|c|}
\hline & Non user & Statin user & p Value \\
\hline Number of participants (N) & 134 & 31 & - \\
\hline Age $(\text { mean } \pm S D)^{*}$ & $46.6 \pm 13.9$ & $60.8 \pm 11.7$ & 0.0001 \\
\hline Range (years) & $18-81$ & $41-86$ & - \\
\hline $\operatorname{Sex}(N(\%)) \dagger$ & & & 0.082 \\
\hline Male & $36(27.1)$ & $13(43.3)$ & \\
\hline Female & 98 (72.9) & $18(56.7)$ & \\
\hline Ethnicity (N (\%))† & & & 0.79 \\
\hline Caucasian & $82(61.2)$ & $17(54.8)$ & \\
\hline African-American & $23(17.2)$ & $8(25.8)$ & \\
\hline Asian/Indian & $14(10.5)$ & $3(9.7)$ & \\
\hline Hispanic & $11(8.2)$ & $1(3.2)$ & \\
\hline Other or not noted & $4(2.9)$ & $2(6.5)$ & \\
\hline BMI $(\text { mean } \pm S D)^{\star} \ddagger$ & $31.2 \pm 8.3$ & $31.7 \pm 6.9$ & 0.73 \\
\hline Range & $19.5-52.4$ & $20.0-50.0$ & \\
\hline Weight $(\text { mean } \pm S D)^{\star} \S$ & $187.9 \pm 51.1$ & $198.5 \pm 43.7$ & 0.24 \\
\hline Smoker (current or past) & $16(11.9 \%)$ & $3(9.7 \%)$ & 0.42 \\
\hline \multicolumn{4}{|l|}{ Lung function (mean $\pm S D)^{*}$} \\
\hline$F E V_{1} \%$ predicted & $75.5 \pm 21.2 \%$ & $67.5 \pm 21.9 \%$ & 0.07 \\
\hline $\mathrm{FEV}_{1}(\mathrm{~L})$ & $2.22 \pm 0.82$ & $1.81 \pm 0.68$ & 0.0052 \\
\hline FVC (L) & $3.02 \pm 0.99$ & $2.56 \pm 0.82$ & 0.0085 \\
\hline $\mathrm{FEF}_{25-75 \%}$ & $1.95 \pm 1.07$ & $1.47 \pm 0.90$ & 0.0125 \\
\hline PEFRף & $369.9 \pm 113.6$ & $347.3 \pm 135.5$ & 0.43 \\
\hline ACT questionnaire score* $($ mean $\pm S D)$ & $15.5 \pm 5.1$ & $16.2 \pm 4.8$ & 0.52 \\
\hline \multicolumn{4}{|l|}{ Peripheral blood counts* (mean $\pm S D)$} \\
\hline WCC $^{* *}$ & $9.06 \pm 2.75$ & $8.43 \pm 2.72$ & 0.34 \\
\hline Absolute eosinophil count ${ }^{\star *}$ & $0.26 \pm 0.24$ & $0.28 \pm 0.27$ & 0.77 \\
\hline Percentage of eosinophil count & $3.14 \pm 2.59$ & $3.38 \pm 2.31$ & 0.68 \\
\hline \multicolumn{4}{|l|}{ Comorbidities (N (\%))† } \\
\hline Atopy/allergies & 79 (58.9) & $17(54.8)$ & 0.675 \\
\hline CAD & $0(0)$ & $5(16.1)$ & $<0.0001$ \\
\hline Hyperlipidaemia & $13(9.5)$ & $29(93.6)$ & $<0.0001$ \\
\hline Diabetes mellitus & $18(13.4)$ & $10(32.3)$ & 0.012 \\
\hline Sinusitis & $19(14.2)$ & $0(0)$ & 0.026 \\
\hline Rhinosinusitis & $98(73.1)$ & $21(67.7)$ & 0.55 \\
\hline GERD & 87 (64.9) & 26 (83.9) & 0.041 \\
\hline \multicolumn{4}{|l|}{ Medications (N (\%))†† } \\
\hline ICS & $134(100)$ & $31(100)$ & - \\
\hline LABA & 107 (79.9) & $25(80.7)$ & 0.90 \\
\hline Albuterol & $117(87.3)$ & $27(87.1)$ & 0.56 \\
\hline Ipratropium & $42(31.3)$ & $15(48.4)$ & 0.023 \\
\hline Systemic corticosteroid use & $23(17.2)$ & $7(22.6)$ & 0.35 \\
\hline Montelukast & $69(51.5)$ & $11(35.5)$ & 0.07 \\
\hline 5-LO inhibitor & $1(0.75)$ & $1(3.2)$ & 0.73 \\
\hline Omalizumab & $4(2.9)$ & $2(6.5)$ & 0.44 \\
\hline Aspirin & $15(11.2)$ & $8(25.8)$ & 0.031 \\
\hline NSAIDs & $8(5.9)$ & $2(6.5)$ & 0.68 \\
\hline PPI or $\mathrm{H}_{2}$-blocker & $63(47.0)$ & $20(64.5)$ & 0.061 \\
\hline
\end{tabular}

*Independent $t$ test used.

†Fisher's exact or $\chi^{2}$ test.

fBMl is in units of $\mathrm{kg} / \mathrm{m}^{2}$.

$\S$ Weight is in units of pounds (lbs).

IPEFR is in units of L/min.

${ }^{\star *}$ WCC and absolute eosinophil count units are in units of $\mathrm{K} / \mathrm{mm}^{3}$.

††Kruskal-Wallis test.

ACT, Asthma Control Test; BMI, body-mass index; CAD, coronary artery disease; FVC, forced vital capacity; GERD, gastro-oesophageal reflux disease; $\mathrm{H}_{2}$, histamine receptor-2; ICS, inhaled corticosteroid; LABA, long-acting $\beta$-agonist; NSAIDs, non-steroidal anti-inflammatory drugs; PEFR, peak expiratory flow rate; PPI, proton-pump inhibitor; 5-LO, 5-lipoxygenase. 
Table 3 Type statins used $(\mathrm{N}=31)$

\begin{tabular}{lll}
\hline Name & Number (\%) & Drug class \\
\hline Simvastatin* & $14(45)$ & Lipophilic \\
Atorvastatin* & $13(42)$ & Lipophilic \\
Lovastatin & $2(6)$ & Lipophilic \\
Pravastatin & $2(6)$ & Hydrophilic \\
\hline *Of all the statins used in patients with severe asthma, $87.1 \%$ \\
were either simvastatin or atorvastatin.
\end{tabular}

ICS use was the best predictor of albuterol use $(p<0.0001)$, and there were no significant interactions among variables. Indicators of typical patient symptoms showed no significant differences (table 1).

\section{Peripheral blood counts}

There were no significant associations between statin use and peripheral eosinophil counts, a systemic marker of allergic inflammation. In the final model, systemic corticosteroid and aspirin use predicted the \% eosinophil count (table 1).

\section{Types of statin used and duration of use}

The majority of our statin users were on simvastatin or atorvastatin ( $45 \%$ vs $42 \%$, table 3 ), which reflects its use in the general US population. Lovastatin and pravastatin were used less frequently $(6 \%)$, and this is also reflected in the general population. The lipophilic statins were used most commonly in our patients.

On average, statin users were on a statin drug for 1.57 \pm 1.32 years, with a median of 1 year of statin use. The range of values was as low as 0.083 years (or 1 month) and as high as 5.25 years (table 4 ).

\section{DISCUSSION}

\section{Summary of main findings}

We evaluated whether statin use in patients with severe asthma was associated with improvement in asthma control and lung function. Our primary finding is that statin use for a median duration of 1 year in this population is associated with a significantly higher ACT score indicating improved asthma symptom control, even when adjusted for potential confounders. We found no statistically significant differences in corticosteroid or rescue bronchodilator use, lung function or peripheral eosinophil counts, although the trends generally favoured statin users.

There have been two recent comprehensive reviews on clinical studies using statins to treat asthma. ${ }^{20} 21$

Table 4 Duration of statin use $(\mathrm{N}=31)$

\begin{tabular}{ll}
\hline Duration of statin use (years)-includes all statins in \\
table 3 \\
\hline Average & $1.57 \pm 1.32$ \\
Median & 1 \\
Range & $0.083-5.25$ \\
\hline
\end{tabular}

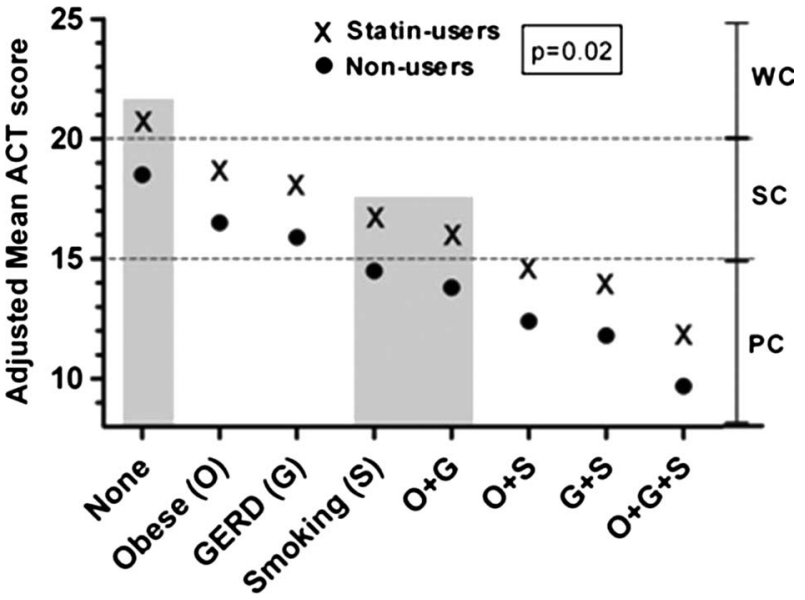

Figure 2 Adjusted mean Asthma Control Test (ACT) score relative to comorbid condition(s). This figure is a graphical representation of what the model predicts in table 1 for ACT scores relative to comorbid conditions. Our analyses showed that severe asthmatics taking statins had higher ACT scores adjusted for and across the spectrum of comorbid conditions ( $\mathrm{O}$, obese; $\mathrm{G}$, gastro-oesophageal reflux disease (GERD); $\mathrm{S}$, history of smoking and 'none' indicates none of these three conditions). The dotted grey lines indicate the degree of asthma control by ACT score: ACT $\geq 20$ indicates well-controlled (WC) asthma, ACT of 19-16 indicates somewhat controlled (SC) asthma and $A C T \leq 15$ indicates poorly controlled (PC) asthma. For patients with a history of smoking (S) and those who are obese with GERD $(\mathrm{O}+\mathrm{G})$, statin use increased ACT score from PC $(\leq 15)$ into the SC range (16-19; area shaded grey). For patients without these comorbidities (none), statin use increased ACT score from SC into the WC $(\geq 20)$ range (area shaded grey). The highest adjusted mean ACT score of 20.7 was observed for statin-using severe asthmatics without these comorbidities (ie, none). At any given comorbid state on the $x$ axis, the difference in the adjusted mean ACT score between a statin user and a non-user is $2.2 \pm 0.94$ points, as indicated in table 1. These adjusted mean ACT values were based on our statistical model using a mean forced vital capacity (FVC) of $2.94 \mathrm{~L}$, the mean value in our study population.

To our knowledge, our study is the first to show a beneficial association between statin use and clinical outcomes in a real-life cohort of severe asthma patients. It is important to stress that our retrospective study design cannot distinguish between association and causation, in particular with the known limitations of statistical modelling. Thus, based on our results, we hypothesise that patients with severe asthma may be a possible target population for treatment with statins, a question ripe for additional investigations.

\section{Statins and biological plausibility in asthma}

Between 2005 and 2008, the Centers for Disease Control and Prevention estimated that $25.1 \pm 0.7 \%$ of Americans aged 45 years and over, approximately 32 million people, used a statin drug. Clinicians and researchers have wondered what effects statins might have on lung 
biology and function, in addition to their potential lung disease modifying effects. In terms of biological plausibility, animal studies utilising allergic mouse models showed that statins have anti-inflammatory, anti-remodelling and immunomodulatory effects that attenuate airway hyper-reactivity and improve lung function. ${ }^{6} 822$ Although less work has been performed on mechanisms of action in lung resident cells, there is evidence of significant action in airway smooth muscle cells, fibroblasts, endothelial cells and epithelial cells in vitro. ${ }^{23}{ }^{24}$ Given the pleiotropic biological effects, widespread use and reasonable safety record of statins, they may be a potential novel therapy for asthma.

\section{Significance of the primary endpoint: the ACT score}

At baseline, the mean ACT scores between non-users and statin users were similar (15.5 vs 16.2 , respectively, $\mathrm{p}=0.52$; table 2). Yet, statin users were on average older, with more comorbidities and had lower lung function (table 2), perhaps indicating a 'sicker' cohort. However, after controlling for confounders in our multivariate analysis, statin users had a significantly higher adjusted mean ACT score than non-users indicating better asthma control ${ }^{17}$ ( $\Delta$ adjusted mean ACT score of 2.2 \pm 0.94 points, $\mathrm{p}=0.02$ (table 1 and figure 2); see Statistical analysis section in the online supplementary material for additional details regarding statistical modelling). As a single measure in the clinic, the ACT score contains significant predictive power for determining asthma risk. Schatz et $a l^{25}$ reported that the minimally important difference (MID) for ACT is a decrease of three points, which confers a $76 \%$ increased risk of short-acting $\beta$-agonist (SABA) use and 33\% increased exacerbations over 12 months. However, even a reduction of two points in the ACT score in their study indicates a $46 \%$ increased risk of SABA use and $21 \%$ increased exacerbations. ${ }^{25}$ By these criteria, our statin users may have gained considerable protection from asthma symptoms and possibly exacerbations over at least 1 year. Our data suggest that patients with severe rather than milder forms of asthma might benefit from statins. However, the therapeutic benefits of statins may occur over many months to years, rather than in the short term.

It is also worth noting that although the adjusted ACT score was higher in statin users, there were no statistically significant improvements in the secondary endpoints (table 1). This discrepancy may be due to the limitations of our sample size or other beneficial yet subtle statin effects captured by ACT and not the singular measures of lung function or medication/inhaler use. The strength of the ACT score is that it has been validated against the asthma specialist's rating of symptom control, quality of life and spirometric lung function. ${ }^{26}$ The weakness of ACT, however, is like any other scoring system or survey in clinical medicine: as a singular measure, it cannot substitute for the entire clinical picture as assessed by the clinician at the bedside. This is especially true when discrepancies appear in the various indices of asthma control in the clinic. Despite this caveat, because of ACT's predictive power (eg, MID as discussed above) in guiding clinical decisions, our findings taken in this light do point us in an important new direction.

\section{Our findings relative to other studies}

Although most of the large retrospective studies in COPD and/or asthma have shown that statins are associated with improved lung function and respiratory outcomes, Ostroukhova and colleagues published a retrospective study showing potential harm. They evaluated statin use in $\mathrm{N}=50$ patients (24 statin exposed and 26 control/unexposed) with mostly mild allergic asthma. ${ }^{27}$ They found that over a span of 6,12 and 24 months, starting statin therapy was associated with reduced $\mathrm{FEV}_{1}$, increased medication use and more symptoms than controls who never started statins. However, it is possible that the statin group may have been sicker at baseline, had worse baseline lung function (ie, $F E V_{1} \%$ predicted not reported) or had a statin indication, such as atherosclerosis, which is known to adversely affect lung function. ${ }^{28} \mathrm{~A}$ multivariate statistical analysis to account for major known confounders was not reported. Notably, the decline in $\mathrm{FEV}_{1}$ in the statin group also showed a proportional decline in the control group over 24 months, with a similar proportional difference in $\mathrm{FEV}_{1}$ between the two groups, raising the possibility that lung function generally worsened in the entire population regardless of statin use. An important difference between our study and Ostroukhova $e t a l$ s study is that they did not report ICS use, an important controller agent that affects asthma outcomes.

Lokhandwala et at evaluated a larger cohort $(\mathrm{N}=479$ statin exposed and 958 unexposed) of patients with asthma on ICS matched for age, sex, race and comorbidities followed for 1 year. They found that asthma-related hospitalisations and/or emergency room visits for patients using statins were approximately half the odds as those not on statins, indicating a benefit to statin use. They used a propensity score-matched study design to account for potential confounders, a strength in their study design. Even after matching, patients on statin therapy had a higher comorbidity index score compared to statin non-users, indicating a potentially sicker population, similar to our cohort. Interestingly, this difference could have biased the findings towards worse outcomes in the statin group. However, the opposite was true, and it is unlikely that this difference in comorbidities could have biased their findings towards a lower risk of asthma-related hospitalisations in the statin group. We speculate that a larger sample size achieving high enough power to assess asthma exacerbations in our UCAN cohort could yield similar results. This idea is supported by another recent study with 3965 patients, which showed an association between statin use and reductions in hospital admissions due to acute asthma exacerbations. ${ }^{3}$

There have been seven published randomised clinical trials using either simvastatin or atorvastatin for the 
treatment of mild or moderate asthma. ${ }^{9-15}$ The most striking difference between these studies and ours is that, besides the study design, we evaluated statin use over a median of 1 year (table 4) in severe asthmatics who were mostly obese females (table 2). Additionally, our population had participants with a known statin indication, including HPL or CAD, important comorbidities that were not studied in these trials. Given that asthma is a chronic disease and that comorbid cardiovascular disease/atherosclerosis may interact adversely with lung function, careful selection of patients without known cardiovascular disease or risk factors, who are statin naïve, who have more severe asthma and who are treated for at least 6-12 months, may be a reasonable approach in designing future randomised clinical trials.

\section{Statins in severe asthma: potential mechanisms and future directions}

The role of obesity or BMI status, systemic inflammation and female sex as features of a statin-responsive population leaves some open questions for further exploration, ${ }^{29}$ especially given what is known about $\mathrm{C}$ reactive protein (CRP) and statins in cardiovascular diseases and obstructive lung disease. ${ }^{28}{ }^{30}$ The links between obesity, systemic and airway inflammation (ie, elevated CRP, leptin and adiponectin), metabolic disease, abnormal lung function in adults and asthma outcomes are an active area of research. ${ }^{29-32}$ Recent data suggest that obesity-associated severe asthma may be a unique clinical phenotype requiring further study. ${ }^{33}$ One potential explanation for why our patients seemed to benefit from statin use despite concurrent ICS/LABA use is that obesity in asthma is associated with reduced response to ICS. $^{34}{ }^{35}$ We speculate that statins may be effective in the obese asthmatic patient who has relative ICS resistance, and who is generally understudied and lacks specific therapy other than weight loss. ${ }^{16}$

It is important to emphasise that $100 \%$ of our patients in this study were using ICS and approximately $80 \%$ used LABAs (table 2). Therefore, statin's observed benefit in our study is potentially additive to standard-of-care controller inhaler therapy. This concept is supported by at least two recent studies by Maneechotesuwan et $a l^{13}$ In their first study, they reported that $10 \mathrm{mg}$ of simvastatin in addition to inhaled budesonide enhanced the anti-inflammatory effects of budesonide (eg, sputum eosinophils), by mechanisms involving alterations in indoleamine 2,3-dioxygenase and interleukin-10 release. In follow-up work, using an in vitro model of asthma, Maneechotesuwan et $a l^{36}$ also found that the combination of simvastatin and fluticasone augments regulatory $\mathrm{T}$ cell (Treg) and Th17 responses to suppress airway inflammation in asthma. Because severe asthma has been linked to Th17 cell and neutrophilic inflammation, ${ }^{37}$ these findings suggest that statins may alter the biology relevant to severe asthma rather than milder disease.

\section{Study limitations}

Our study is a retrospective, cross-sectional study which can reveal associations. It is a relatively small study $(\mathrm{N}=165)$ that was performed in our severe asthma clinic at one academic medical centre. A retrospective design cannot account for all potential confounders and limits final conclusions. Of note, the statin users in our study may be fundamentally different from non-users, namely that all patients taking statins had CAD and/or HPL, despite our efforts at designing the best statistical models to account for such confounders. Also, we did not assess the effects of corticosteroid use on lipid or glucose metabolism that may have important interactions and confound our results. Statistical corrections for missing data were beyond the scope of this work; this remains a drawback of most retrospective studies.

\section{CONCLUSIONS}

In conclusion, we report that statin use was associated with improved asthma control in patients with severe asthma. We speculate that adult asthmatics with poor asthma control and atherosclerosis or systemic inflammation may be a higher risk group worthy of further study. We believe that more work is needed to (1) determine whether a subpopulation of asthma exists that might benefit from statins, (2) investigate whether systemic inflammation and/or atherosclerosis/CAD in asthma represent a subphenotype that is more refractory to standard treatment and (3) determine whether the obese patient with severe asthma may be a reasonable candidate for future statin clinical trials.

\section{Author affiliations}

${ }^{1}$ Department of Internal Medicine, University of California, Davis School of Medicine, Sacramento, California, USA

${ }^{2}$ Division of Pulmonary, Critical Care Medicine, and Sleep Medicine, University of California, Davis School of Medicine, Sacramento, California, USA ${ }^{3}$ Clinical and Translational Science Center, University of California, Davis School of Medicine, Sacramento, California, USA

${ }^{4}$ Center for Comparative Respiratory Biology \& Medicine, University of California, Davis School of Medicine, Sacramento, California, USA ${ }^{5}$ Department of Public Health Sciences, Division of Biostatistics, University of California, Davis School of Medicine, Sacramento, California, USA

${ }^{6}$ Department of Obstetrics \& Gynecology, University of California, Davis School of Medicine, Sacramento, California, USA

Acknowledgements The authors would like to thank Professor Samuel Louie for inspiring them to utilise the University of California, Davis Asthma Network (UCAN) severe asthma cohort in their studies, the countless helpful discussions regarding the management of severe asthma, and his reading of this manuscript.They also acknowledge the contributions and work of the registered respiratory therapists Claudia Vukovich and Celeste Kivler in helping them develop and manage the severe asthma database. The authors would also like to thank Kent Anderson and Larry Errecart of the University of California (UC) Davis Clinical and Translational Science Center (CTSC) Biomedical Informatics core for their help in developing and refining the online Access database.

Contributors AAZ conceived of the project along with JAL. AAZ codesigned the database with AP, collected and analysed the data with MW, developed the tables and figures, and wrote the manuscript. $\mathrm{JO}$ helped in collecting the data, entering it into the online database and writing parts of the Methods and 
Materials section. MW as the primary biostatistician on the project helped analyse and organise the data as presented. OF and VG helped in collecting the data, entering it into the online database and writing sections of the Discussion. ML helped in doing the initial statistical analyses, power analyses, and in how the data should be collected and organised in the database. AL helped in collecting the data, entering it into the online database and writing sections of the Results. AP helped AAZ in codesigning the study online database, integrating it with the electronic medical record and pre-existing UCAN database, and automating the data collection, storage, integrity and security.. He also provided programming to query the database and collect it in an Excel format. JAL provided initial and continuous guidance regarding the study design, data analysis, and helped write and edit the final version of the manuscript. NJK helped AAZ with the experimental and database designing. He also provided guidance throughout the project, interpreted the data, and helped write and edit the final version of the manuscript.

Funding This research was supported by the National Center for Advancing Translational Sciences, National Institutes of Health $(\mathrm{NIH})$, through grant \#UL1 TR000002 to AAZ and NJK; CTSC NIH KL2 (K12) Award TR000134 to AAZ; NIH/NHLBI T32 Training Grant \#HL07013 to AAZ; and the NIH K30 Mentored Clinical Research Training Program (MCRTP) to AAZ, through grant \#UL1 RR024146; and NIH HL105573 to NJK.

Competing interests None.

Ethics approval UC Davis Institutional Review Board (IRB; Expedited Review Committee, protocol \#200715026).

Provenance and peer review Not commissioned; externally peer reviewed.

Data sharing statement No additional data are available.

Open Access This is an Open Access article distributed in accordance with the Creative Commons Attribution Non Commercial (CC BY-NC 3.0) license, which permits others to distribute, remix, adapt, build upon this work noncommercially, and license their derivative works on different terms, provided the original work is properly cited and the use is non-commercial. See: http:// creativecommons.org/licenses/by-nc/3.0/

\section{REFERENCES}

1. Levine SJ, Wenzel SE. Narrative review: the role of Th2 immune pathway modulation in the treatment of severe asthma and its phenotypes. Ann Intern Med 2010;152:232-7.

2. Lokhandwala T, West-Strum D, Banahan BF, et al. Do statins improve outcomes in patients with asthma on inhaled corticosteroid therapy? A retrospective cohort analysis. BMJ 2012;2:1-8.

3. Huang CC, Chan WL, Chen YC, et al. Statin use in patients with asthma: a nationwide population-based study. Eur J Clin Invest 2011;41:507-12.

4. Alexeeff SE, Litonjua AA, Sparrow D, et al. Statin use reduces decline in lung function: VA Normative Aging Study. Am J Respir Crit Care Med 2007;176:742-7.

5. Blamoun Al, Batty GN, DeBari VA, et al. Statins may reduce episodes of exacerbation and the requirement for intubation in patients with COPD: evidence from a retrospective cohort study. Int J Clin Pract 2008;62:1373-8.

6. Zeki A, Franzi L, Last J, et al. Simvastatin inhibits airway hyperreactivity: implications for the mevalonate pathway and beyond. Am J Respir Crit Care Med 2009;180:731-40.

7. Takeda N, Kondo M, Ito S, et al. Role of RhoA inactivation in reduced cell proliferation of human airway smooth muscle by simvastatin. Am J Respir Cell Mol Biol 2006;35:722-9.

8. McKay A, Leung BP, Mclnnes IB, et al. A novel anti-inflammatory role of simvastatin in a murine model of allergic asthma. $J$ Immunol 2004;172:2903-8

9. Moini A, Azimi G, Farivar A. Evaluation of atorvastatin for the treatment of patients with asthma: a double-blind randomized clinical trial. Allergy Asthma Immunol Res 2012;4:290-4.

10. Braganza G, Chaudhuri R, McSharry C, et al. Effects of short-term treatment with atorvastatin in smokers with asthma-a randomized controlled trial. BMC Pulm Med 2011;11:16.

11. Cowan DC, Cowan JO, Palmay R, et al. Simvastatin in the treatment of asthma: lack of steroid-sparing effect. Thorax 2010;65:891-6.

12. Hothersall EJ, Chaudhuri R, McSharry C, et al. Effects of atorvastatin added to inhaled corticosteroids on lung function and sputum cell counts in atopic asthma. Thorax 2008;63:1070-5.
13. Maneechotesuwan K, Ekjiratrakul W, Kasetsinsombat K, et al. Statins enhance the anti-inflammatory effects of inhaled corticosteroids in asthmatic patients through increased induction of indoleamine 2, 3-dioxygenase. J Allergy Clin Immunol 2010;126:754-762.e1.

14. Menzies D, Nair A, Meldrum KT, et al. Simvastatin does not exhibit therapeutic anti-inflammatory effects in asthma. $J$ Allergy Clin Immunol 2007;119:328-35.

15. Fahimi $F$, Salamzadeh J, Jamaati $H$, et al. Do statins improve lung function in asthmatic patients? A randomized and double-blind trial Iran J Pharm Sci 2009;5:13-20.

16. Moore WC, Meyers D, Wenzel SE, et al. Identification of asthma phenotypes using cluster analysis in the Severe Asthma Research Program. Am J Respir Crit Care Med 2010;181:315-23.

17. Nathan R, Sorkness C, Kosinski M, et al. Development of the asthma control test: a survey for assessing asthma control. J Allergy Clin Immunol 2004;113:59-65.

18. Schatz M, Sorkness C, Li JT, et al. Asthma Control Test: reliability, validity, and responsiveness in patients not previously followed by asthma specialists. J Allergy Clin Immunol 2006;117: 549-56.

19. Ko FWS, Leung T-F, Hui DSC, et al. Asthma Control Test correlates well with the treatment decisions made by asthma specialists. Respirology 2009;14:559-66.

20. Yuan C, Zhou L, Cheng J, et al. Statins as potential therapeutic drug for asthma? Respir Res 2012;13:108.

21. Silva $D$, Couto $M$, Delgado $L$, et al. A systematic review of statin efficacy in asthma. J Asthma 2012;49:885-94.

22. Zeki AA, Bratt JM, Rabowsky M, et al. Simvastatin inhibits goblet cell hyperplasia and lung arginase in a mouse model of allergic asthma: a novel treatment for airway remodeling? Transl Res 2010;156:335-49.

23. Iwata $A$, Shirai $\mathrm{R}$, Ishii $\mathrm{H}$, et al. Inhibitory effect of statins on inflammatory cytokine production from human bronchial epithelia cells. Clin Exp Immunol 2012;168:234-40.

24. Zeki AA, Thai $P$, Kenyon NJ, et al. Differential effects of simvastatin on IL-13-induced cytokine gene expression in primary mouse tracheal epithelial cells. Respir Res 2012;13:38.

25. Schatz M, Kosinski M, Yarlas AS, et al. The minimally important difference of the Asthma Control Test. J Allergy Clin Immunol 2009;124:719-23.e1.

26. Reddel HK, Taylor DR, Bateman ED, et al. An Official American Thoracic Society/European Respiratory Society Statement: asthma control and exacerbations. Am J Respir Crit Care Med 2009;180:59-99.

27. Ostroukhova M, Kouides RW, Friedman E. The effect of statin therapy on allergic patients with asthma. Ann Allergy Asthma Immunol 2009;103:463-8.

28. Van Eeden S, Leipsic J, Paul Man SF, et al. The relationship between lung inflammation and cardiovascular disease. Am J Respir Crit Care Med 2012;186:11-16

29. Scott HA, Gibson PG, Garg ML, et al. Airway inflammation is augmented by obesity and fatty acids in asthma. Eur Respir $J$ 2011;38:594-602.

30. Melbye H, Halvorsen DS, Hartz I, et al. Bronchial airflow limitation, smoking, body mass index, and statin use are strongly associated with the C-reactive protein level in the elderly. The Troms $ø$ Study 2001. Respir Med 2007;101:2541-9.

31. Sutherland TJT, Cowan JO, Young S, et al. The association between obesity and asthma: interactions between systemic and airway inflammation. Am J Respir Crit Care Med 2008;178:469-75.

32. Zeki A, Kenyon NJ, Goldkorn T. Statin drugs, metabolic pathways, and asthma: a therapeutic opportunity needing further research. Drug Metab Lett 2011;5:40-4.

33. Gibeon D, Batuwita K, Osmond M, et al. Obesity-associated severe asthma represents a distinct clinical phenotype: analysis of the British Thoracic Society Difficult Asthma Registry Patient Cohort according to BMI. Chest 2013;143:406-14.

34. Telenga ED, Tideman SW, Kerstjens HAM, et al. Obesity in asthma: more neutrophilic inflammation as a possible explanation for a reduced treatment response. Allergy 2012;67:1060-8.

35. Boulet L-P, Franssen $E$. Influence of obesity on response to fluticasone with or without salmeterol in moderate asthma. Respir Med 2007;101:2240-7.

36. Maneechotesuwan K, Kasetsinsombat K, Wamanuttajinda V, et al. Statins enhance the effects of corticosteroids on the balance between regulatory T cells and Th17 cells. Clin Exp Allergy 2013;43:212-22.

37. Nakagome K, Matsushita S, Nagata M. Neutrophilic inflammation in severe asthma. Int Arch Allergy Immunol 2012;158(Suppl 1):96-102. 